\title{
Editorial: The Role of Turbulence in the Solar Wind, Magnetosphere, Ionosphere Dynamics
}

\author{
Marina Stepanova ${ }^{1,2 *}$, Joseph E. Borovsky ${ }^{3}$, Alessandro Retino ${ }^{4}$, Vadim Uritsky ${ }^{5,6}$, \\ Zoltán Vörös ${ }^{7}$ and Gaetano Zimbardo ${ }^{8,9}$
}

${ }^{1}$ Physics Department, University of Santiago of Chile (USACH), Santiago, Chile, ${ }^{2}$ Center for Interdisciplinary Research in Astrophysics and Space Sciences, University of Santiago of Chile (USACH), Santiago, Chile, ${ }^{3}$ Space Science Institute, Boulder, CO, United States, ${ }^{4}$ Laboratoire de Physique des Plasmas, CNRS, Observatoire de Paris, Sorbonne Université, Université ParisSaclay, École Polytechnique, Institut Polytechnique de Paris, Palaiseau, France, ${ }^{5}$ Department of Physics, The Catholic University of America, Washington, DC, United States, ${ }^{6}$ NASA Goddard Space Flight Center, Greenbelt, MD, United States, ${ }^{7}$ Space Research Institute, Austrian Academy of Sciences Graz, Graz, Austria, ${ }^{8}$ Dipartimento di Fisica, Universitá della Calabria, Rende, Italy, ${ }^{9}$ Istituto Nazionale di Astrofisica, Sezione INAF Presso l'Universitá della Calabria, Rende, Italy

Keywords: turbulence, space plasmas, heliosphere, solar wind, magnetosphere

Editorial on the Research Topic

The Role of Turbulence in the Solar Wind, Magnetosphere, Ionosphere Dynamics

This Research Topic is dedicated to one of the most intriguing and least understood phenomena of space plasma physics: nearly collisionless turbulence. Even at the beginning of space exploration, there was evidence that space plasmas might be turbulent. For example, Ness et al. (1964) analyzed the magnetic field measurements made by the IMP 1 satellite and reported the existence of the region of turbulence between the magnetopause and the bow shock now known as the magnetosheath. Later on, Fairfield and Ness (1970) calculated the power-law spectra of magnetic fluctuations in the magnetosheath using IMP 4 measurements. A highly fluctuating magnetic field in the geomagnetic tail was observed by the IMP 1 Hruška and Hrušková (1969) and IMP 3 Hruška and Hrušková (1970) satellites. Coleman (1968) analyzed the magnetic fluctuations in the solar wind using the data from the Mariner 2 spacecraft. The obtained power spectral density of these fluctuations closely resembled a typical turbulent spectrum. More sophisticated space missions were launched during the following decades, giving more insight about the main characteristics of space plasmas, including fluctuations of all plasma macro-parameters and fields. Nonetheless, turbulence is only one possible interpretation of these fluctuations, and the discussion about what we observe continues. This Research Topic contains different, sometimes even contradictory, points of view on the nature of turbulence and observed phenomena. It covers eight reviews and three mini-reviews, providing the reader with a view of the current state of this field. It also includes original research and prospective papers.

Four papers in this Research Topic are focused on determining what is turbulence and what is mistaken for turbulence. In a perspective article, Klimchuk and Antiochos argue that in the closed corona of the Sun the ubiquitous current sheets arise from the quasi-static twisting of flux tubes rather than from the action of dynamic turbulence. These currents sheets, being the sites of reconnection (nanoflares), could play a key role in the heating of the corona. Similarly, the review article by Borovsky argues that much of the structures (including current sheets) in the solar wind are also not turbulence: his review examines the properties of the structure of the solar wind and the arguments for certain structure types having their origin in the corona. doi: $10.3389 /$ fspas.2021.763190
Viall et al. extensively review solar observations, solar-wind measurements, and computer 
simulations to explore the issue of what structures in the solar wind are created at the Sun and what structures are created in the wind away from the Sun. Viall et al. assess the current knowledge and outline future steps for progress. Borovsky and Mina perform side-by-side analyses of the measurements of fluctuations in (1) the Navier-Stokes turbulence of a wind tunnel and (2) the Alfvénic solar wind, focusing on the similarities and differences in the statistical and the physical properties of the fluctuations. The question raised is: Why do the Alfvénic fluctuations statistically look like turbulence?

Our Research Topic also includes a set of works dedicated to the study of different types of turbulence in the heliosphere. In particular, Wawrzaszek and Echim concentrate on the study of turbulence intermittency, an important diagnostics of turbulent energy cascade, throughout the heliosphere. Various indicators of intermittency are reviewed, and it is found that intermittency increases with radial distance in Alfvénic solar wind, that the intermittency of fast solar wind at solar minimum decreases with latitude, while at solar maximum the intermittency values are more scattered. Boldyrev et al. review several fluid models for fluctuations in low-to-moderate beta plasmas, which are representative of space plasma environments such as the Earth's magnetosphere and magnetosheath as well as the solar corona. The general physical derivation of the model equations is presented and three special cases are treated in detail, including the derivation of the dispersion relations for linear modes as well as the conserved quantities. Assumptions and limits of applicability of each model are discussed. Guo et al. address the fact that the presence of solar-wind turbulence leads to the rippling of interplanetary-shock surfaces. The role of preexisting upstream turbulence and shock surface rippling in enhancing the acceleration of particles is emphasized, and it is proposed that this may help to solve the injection problem of shock acceleration, in particular at quasi-perpendicular shocks. Pitna et al. point out that while particle acceleration at shocks in the presence of turbulence has been long considered, only recently has the evolution of turbulence itself from upstream to downstream received enough attention. They show that, compared to upstream, the downstream turbulent power in magnetic field, velocity, and density is enhanced by about one order of magnitude, and that downstream turbulence is always more compressive. Both the papers of Guo et al. and Pitna et al. point out that in-situ observations of interplanetary shocks provide an invaluable opportunity to understand how shocks interact with large-scale turbulence and accelerate energetic particles.

Smith and Vasquez review the evidence for multi-scale turbulent in-situ dynamics that results in the heating of the solar wind. The generation, transport and dissipation of fluctuation energy are critically examined offering a wide discussion of the often controversial scenarios for energy transfer across scales. Perhaps some fundamental questions remain unanswered because of the lack of multi-point measurements and realistic simulations. Roberts et al. investigated intermittency of turbulent electron density fluctuations in the solar wind at sub-ion scales using calibrated spacecraft potential from the Magnetospheric MultiScale mission.
The scale-dependent kurtosis is found to increase towards ion scales. Then it plateaus through the sub-ion range. However, discrepancies between statistics of temporal and spatial fluctuation exist, for which several alternative explanations are offered. Based on the linear Vlasov theory, Narita et al. examined the plasma dielectric tensor deriving analytic expressions for transport ratios and scaling laws for the electric and magnetic fields. Fluid-scale properties of the kinetic Alfvén mode are derived from the kinetic treatment of the dielectric tensor. The developed methods are useful for the wave mode identification in spacecraft observations. D'Amicis et al. review recent developments on the complexity of solar wind magnetosphere interactions with the emphasis on solar wind turbulence driven geomagnetic response. The role played by Alfvenic turbulence in the solar wind-magnetosphere coupling is accentuated. It is also emphasized that the geomagnetic response is affected by internal magnetospheric conditions and by the total energy of solar wind fluctuations. Moya and Navarro used quasi-linear kinetic theory to investigate the effects of a turbulence background spectrum on the relaxation of the ion-cyclotron temperature anisotropy instability in the solar wind. Different initial levels of turbulence amplitude and spectral shapes are considered. It is shown that all spectrum shapes can heat protons preferentially in the direction perpendicular to the ambient magnetic field, even if the plasma is initially stable. Possible implications for recent Parker Solar Probe data are discussed.

Turbulence in the magnetosheath is reviewed by Rakhmanova et al. In particular, they discuss the applicability of the Taylor hypothesis, the main features of the spectra of plasma, magnetic fluctuations fitted by power laws for various MHD and kinetic regimes, as well as the intermittent character of turbulence. Of special interest is the study of the plasma fluctuations measured with sufficient time resolution to explore plasma turbulence at kinetic scales, along with the influence of the solar wind turbulence and geometry of the bow shock to the development of the magnetosheath turbulence.

Antonova and Stepanova review the paradigm of the geomagnetic tail as a turbulent wake behind an obstacle and its application to the magnetospheric dynamics. In particular, they examine the role of turbulence on the MHD scales in the plasma transport and mixing, and the stability of the turbulent plasma sheet. Based on their findings, the large-scale two-vortex magnetospheric convection generated by the magnetospheric plasma pressure gradients is proposed as a source of energy for turbulence development and possibly the driving or integral vortex of such turbulence. The turbulent flows in the magnetotail appear during the 3D MHD simulations of the global magnetosphere. Meanwhile, a review by El-Alaoui et al. describes a method to couple the large-scale drivers of MHD turbulence to the local kinetic scales. The obtained power spectral density of magnetic fluctuations is consistent with observations of turbulence in the magnetotail. The authors highlight the role of resistivity as a source of dissipation that contributes to plasma sheet turbulence in two ways. First, on a large scale, it causes reconnection, which drives the turbulent flows, and secondly, it dissipates the energy on smaller scales. They also consider the influence of the Kelvin-Helmholtz instability along the flank boundaries. According to El-Alaoui et al. 
turbulent acceleration might lead to the appearance of non-thermal tails in plasma distributions (a kappa distribution). Eyelade et al. established a relation between the turbulent eddy diffusion and kappa indexes for different values of plasma $\beta$ parameter in the Earth's plasma sheet. They found that several processes related to MHD turbulence lead to either an increase or decrease of the $\kappa$ index of kappa distribution functions, depending on the value of $\beta$ and the direction of the turbulent transport with respect to the plasma sheet.

Studies of turbulent processes in the ionosphere are presented in a number of works. The mini-review by Akbari et al. focuses on Langmuir waves and turbulence in the auroral ionosphere caused by magnetospheric electron beams. The review provides a concise but rigorous theoretical description of the Langmuir wave mechanism, and a summary of many years of observations of this phenomenon using spaceborne and ground-based instruments. The importance of conjugate multi-instrument campaigns involving in situ wave and particle measurements and incoherent scatter radar data is emphasized. Turbulence in collisionless space plasmas is known to be a major cause of the anomalous transport and resistivity. The paper by Guio and Pecseli addresses these important phenomena, paying particular attention to cross-scale wave mechanisms that contribute to the anomalous resistivity and diffusion. Intermittent kinetic structures able to limit the free flow of charged plasma particles (such as electron and ion phase space vortices) are also discussed. The perspective article by Chaston is dedicated to transient multiscale structures in the auroral acceleration region playing a major part in shaping auroral displays. The author emphasizes the importance of coordinated in-situ and ground based observations allowing one to relate the nonlinear forcing of the plasma above auroral arcs with visible auroral forms. The examples presented in the paper provide evidence for a turbulent cascade inside the auroral acceleration region, likely supported by large-amplitude inertial Alfvén waves.

There are a few works dedicated to the turbulence in the magnetospheres of the outer planets and some fundamental aspects of space plasmas. In particular, Saur discusses plasma turbulence at outer planets where conditions are different from those in the solar wind, e.g., existence of a strong planetary

\section{REFERENCES}

Coleman, P. J. J., Jr (1968). Turbulence, Viscosity, and Dissipation in the SolarWind Plasma. Astrophysical J. 153, 371. doi:10.1086/149674

Fairfield, D. H., and Ness, N. F. (1970). Magnetic Field Fluctuations in the Earth's Magnetosheath. J. Geophys. Res. 75 (31), 6050-6060. doi:10.1029/ ja075i031p06050

Hruška, A., and Hrušková, J. (1969). Long Time-Scale Magnetodynamic Noise in the Geomagnetic Tail. Planet. Space Sci. 17 (8), 1497-1504.

Hruška, A., and Hrušková, J. (1970). Transverse Structure of the Earth's Magnetotail and Fluctuations of the Tail Magnetic Field. J. Geophys. Res. 75 (13), 2449-2457.

Ness, N. F., Scearce, C. S., and Seek, J. B. (1964). Initial Results of the Imp 1 Magnetic Field experiment. J. Geophys. Res. 69 (17), 3531-3569. doi:10.1029/ jz069i017p03531 magnetic field, stronger plasma inhomogeneities and larger abundance of heavy ions. Spacecraft observations at Jupiter and Saturn are reviewed, namely, in the magnetosphere, magnetosheath and at moons, and implications for plasma heating and particle acceleration are discussed. The need for new measurements from future missions, in particular at Uranus and Neptune, is discussed.

As shown in this editorial, past and present satellite missions have provided us with an unprecedented opportunity to study the turbulence in space plasmas in situ. As a result, the heliosphere became the world's largest laboratory for studying various types of plasma turbulence. The majority of turbulent properties obtained are universal and relevant not only for space physics systems but also for other astrophysical objects with similar turbulent processes. We believe that this Research Topic will enable space scientists and astrophysicists, particularly those in their early careers, to understand a variety of aspects of a wide range of sometimes controversial topics and arrive at their own conclusions.

\section{AUTHOR CONTRIBUTIONS}

All authors listed have made a substantial, direct and intellectual contribution to the work, and approved it for publication.

\section{FUNDING}

MS was supported by the FONDECYT 1211144 and AFOSR $\mathrm{N}^{\circ}$ FA9550-19-1-0384 research grants, JB was supported by the NSF SHINE program, ZV was supported by the Austrian FWF projects P28764-N27.

\section{ACKNOWLEDGMENTS}

MS thanks Elizaveta Antonova for helpful conversations.

Conflict of Interest: The authors declare that the research was conducted in the absence of any commercial or financial relationships that could be construed as a potential conflict of interest.

Publisher's Note: All claims expressed in this article are solely those of the authors and do not necessarily represent those of their affiliated organizations, or those of the publisher, the editors, and the reviewers. Any product that may be evaluated in this article, or claim that may be made by its manufacturer, is not guaranteed or endorsed by the publisher.

Copyright (C) 2022 Stepanova, Borovsky, Retino, Uritsky, Vörös and Zimbardo. his is an open-access article distributed under the terms of the Creative Commons Attribution License (CC BY). The use, distribution or reproduction in other forums is permitted, provided the original author(s) and the copyright owner(s) are credited and that the original publication in this journal is cited, in accordance with accepted academic practice. No use, distribution or reproduction is permitted which does not comply with these terms. 